\title{
On f-rectifying curves in the Euclidean 4-space
}

\author{
Zafar Iqbal \\ Department of Mathematics, \\ Kaliyaganj College, Kaliyaganj, \\ Uttar Dinajpur - 733 129, \\ West Bengal, India \\ email: zafariqbal_math@yahoo.com
}

\author{
Joydeep Sengupta \\ Department of Mathematics and \\ Statistics, Aliah University, \\ Newtown, Kolkata - 700 160, \\ West Bengal, India \\ email: joydeep1972@yahoo.com
}

\begin{abstract}
A rectifying curve in the Euclidean 4-space $\mathbb{E}^{4}$ is defined as an arc length parametrized curve $\gamma$ in $\mathbb{E}^{4}$ such that its position vector always lies in its rectifying space (i.e., the orthogonal complement $\mathrm{N}_{\gamma}{ }^{\perp}$ of its principal normal vector field $\mathrm{N}_{\gamma}$ ) in $\mathbb{E}^{4}$. In this paper, we introduce the notion of an f-rectifying curve in $\mathbb{E}^{4}$ as a curve $\gamma$ in $\mathbb{E}^{4}$ parametrized by its arc length $s$ such that its $f$-position vector $\gamma_{f}$, defined by $\gamma_{f}(s)=\int f(s) d \gamma$ for all $s$, always lies in its rectifying space in $\mathbb{E}^{4}$, where $f$ is a nowhere vanishing integrable function in parameter $s$ of the curve $\gamma$. Also, we characterize and classify such curves in $\mathbb{E}^{4}$.
\end{abstract}

\section{Introduction}

Let $\mathbb{E}^{3}$ denote the Euclidean 3-space. Let $\gamma: \mathrm{I} \longrightarrow \mathbb{E}^{3}$ be a unit-speed curve (parametrized by arc length $s$ ) with at least four continuous derivatives. It is needless to mention that I denotes a non-trivial interval in $\mathbb{R}$, i.e., a connected set in $\mathbb{R}$ containing at least two points. For the curve $\gamma$ in $\mathbb{E}^{3}$, we consider the Frenet apparatus $\left\{\mathrm{T}_{\gamma}, \mathrm{N}_{\gamma}, \mathrm{B}_{\gamma}, \mathrm{K}_{\gamma}, \tau_{\gamma}\right\}$, where $\mathrm{T}_{\gamma}=\gamma^{\prime}$ is the unit tangent vector field of $\gamma, N_{\gamma}$ is the unit principal normal vector field of $\gamma$ obtained by

2010 Mathematics Subject Classification: 53A04, 53C40

Key words and phrases: Euclidean 4-space, Frenet formulae, rectifying curve, curvature 
normalizing the acceleration vector field $\mathrm{T}_{\gamma}^{\prime}, \mathrm{B}_{\gamma}=\mathrm{T}_{\gamma} \times \mathrm{N}_{\gamma}$ is the unit binormal vector field of the curve $\gamma$ so that the Frenet frame $\left\{T_{\gamma}, N_{\gamma}, B_{\gamma}\right\}$ is positive definite along $\gamma$ having the same orientation as that of $\mathbb{E}^{4}$, and $\kappa_{\gamma}: \mathrm{I} \longrightarrow \mathbb{R}$ is at least twice differentiable function with $\kappa_{\gamma}>0$, known as the curvature of $\gamma$, and $\tau_{\gamma}: \mathrm{I} \longrightarrow \mathbb{R}$ is a differentiable function, called the torsion of the curve $\gamma$. Then the Frenet formulae for the curve $\gamma$ are given by $([1,2])$

$$
\left(\begin{array}{c}
\mathrm{T}_{\gamma}^{\prime} \\
\mathrm{N}_{\gamma}^{\prime} \\
\mathrm{B}_{\gamma}^{\prime}
\end{array}\right)=\left(\begin{array}{ccc}
0 & \mathrm{~K}_{\gamma} & 0 \\
-\mathrm{K}_{\gamma} & 0 & \tau_{\gamma} \\
0 & -\tau_{\gamma} & 0
\end{array}\right)\left(\begin{array}{c}
\mathrm{T}_{\gamma} \\
\mathrm{N}_{\gamma} \\
\mathrm{B}_{\gamma}
\end{array}\right) .
$$

The planes spanned by $\left\{\mathrm{T}_{\gamma}, \mathrm{N}_{\gamma}\right\},\left\{\mathrm{N}_{\gamma}, \mathrm{B}_{\gamma}\right\}$ and $\left\{\mathrm{T}_{\gamma}, \mathrm{B}_{\gamma}\right\}$ are called the osculating plane, the normal plane and the rectifying plane of the curve $\gamma$, respectively (cf. $[1,2,3])$.

In the Euclidean 3-space $\mathbb{E}^{3}$, the notion of a rectifying curve was introduced by B.Y. Chen in [3] as a tortuous curve whose position vector always lies in the rectifying plane of the curve. That is, for a rectifying curve $\gamma: \mathrm{I} \longrightarrow \mathbb{E}^{3}$, the position vector of $\gamma$ can be expressed as

$$
\gamma(s)=\lambda(s) \mathrm{T}_{\gamma}(s)+\mu(s) \mathrm{B}_{\gamma}(\mathrm{s}), \quad s \in \mathrm{I},
$$

for two unique smooth functions $\lambda, \mu: I \longrightarrow \mathbb{R}$.

Several characterizations and classification of the rectifying curves in $\mathbb{E}^{3}$ were studied in $[3,4,5,6]$. Meanwhile, the notion of rectifying curves were extended to several sorts of Riemannian and pseudo-Riemannian spaces. As for example, many interesting characterizations and classification of rectifying curves in the higher dimensional Euclidean spaces were studied in $[7,8]$, and the same in Minkowski 3-space $\mathbb{E}_{1}^{3}$ were studied in $[9,10]$.

In [7], a rectifying curve in the Euclidean 4 -space $\mathbb{E}^{4}$ was defined as a curve $\gamma: \mathrm{I} \longrightarrow \mathbb{E}^{4}$ parametrized by its arc length $\mathrm{s}$ such that its position vector always lies in its rectifying space, i.e., in the orthogonal complement $\mathrm{N}_{\gamma}{ }^{\perp}$ of its principal normal vector field $\mathrm{N}_{\gamma}$. In collateral to this, in this paper, we introduce the notion of an f-rectifying curve in $\mathbb{E}^{4}$ as a curve $\gamma: \mathrm{I} \longrightarrow \mathbb{E}^{4}$ parametrized by its arc length $s$ such that its $f$-position vector, denoted and defined by $\gamma_{f}(s):=\int f(s) d \gamma$ for all $s \in I$, always lies in its rectifying space. Here $f: I \longrightarrow \mathbb{R}$ is a nowhere vanishing integrable function in arc length parameter $s$ of the curve $\gamma$. In this regard, let us mention that non-null and null f-rectifying curves were investigated in Minkowski 3-space $\mathbb{E}_{1}^{3}[11,12]$ and null f-rectifying curves were explored in Minkowski space-time $\mathbb{E}_{1}^{4}$ [13]. 
In the first section, we give requisite basic preliminaries and then introduce the notion of f-rectifying curves in $\mathbb{E}^{4}$. Thereafter, the second section is devoted to investigate some geometric characterizations of f-rectifying curves in $\mathbb{E}^{4}$. In the concluding section, we attempt for some classification of $f$-rectifying curves in terms of their f-position vectors in $\mathbb{E}^{4}$. Finally, we cite an example of an f-rectifying curve lying wholly in $\mathbb{E}^{4}$. This is how this paper is organised.

\section{Preliminaries}

The Euclidean 4-space $\mathbb{E}^{4}$ is the four dimensional real vector space $\mathbb{R}^{4}$ equipped with the standard inner product $\langle\cdot, \cdot\rangle$ defined by

$$
\langle v, w\rangle:=\sum_{i=1}^{4} v_{i} w_{i}
$$

for all vectors $v=\left(v_{1}, v_{2}, v_{3}, v_{4}\right), w=\left(w_{1}, w_{2}, w_{3}, w_{4}\right) \in \mathbb{R}^{4}$. As usual, the norm or length of a vector $v=\left(v_{1}, v_{2}, v_{3}, v_{4}\right) \in \mathbb{R}^{4}$ is denoted and defined by

$$
\|v\|:=\sqrt{\langle v, v\rangle}=\sqrt{\sum_{i=1}^{4} v_{i}^{2}} .
$$

Let $\gamma: \mathrm{J} \longrightarrow \mathbb{E}^{4}$ be an arbitrary smooth curve in $\mathbb{E}^{4}$ parametrized by $\mathrm{t}$ and $\gamma^{\prime}$ stands for its velocity vector field. If we change the parameter $t$ by arc length function $s=s(t)$ based at some $t_{0} \in J$ given by $s(t)=\int_{t_{0}}^{t}\left\|\gamma^{\prime}(u)\right\| d u$ such that $\left\langle\gamma^{\prime}(s), \gamma^{\prime}(s)\right\rangle=1$ for all possible $s$, then $\gamma$ is a curve in $\mathbb{E}^{4}$ parametrized by arc length $s$ or a unit-speed curve in $\mathbb{E}^{4}$. We may assume that $\gamma$ is at least 4-times continuously differentiable. Now, let $\mathrm{T}_{\gamma}, \mathrm{N}_{\gamma}, \mathrm{B}_{\gamma_{1}}$ and $\mathrm{B}_{\gamma_{2}}$ denote the unit tangent vector field, the unit principal normal vector field, the unit first binormal vector field and the unit second binormal vector field of the curve $\gamma$ in $\mathbb{E}^{4}$, respectively, so that for each $s \in I$, the set $\left\{T_{\gamma}(s), N_{\gamma}(s), B_{\gamma_{1}}(s), B_{\gamma_{2}}(s)\right\}$ forms an orthonormal basis for $\mathbb{E}^{4}$ at the point $\gamma(s)$. Also, let $\kappa_{\gamma_{1}}, \kappa_{\gamma_{2}}$ and $\kappa_{\gamma_{3}}$ denote the first curvature, the second curvature and the third curvature of the curve $\gamma$ in $\mathbb{E}^{4}$, respectively. Thus $\left\{\mathrm{T}_{\gamma}, \mathrm{N}_{\gamma}, \mathrm{B}_{\gamma_{1}}, \mathrm{~B}_{\gamma_{2}}\right\}$ is the dynamic Frenet frame along the curve $\gamma$ having the same orientation as that of $\mathbb{E}^{4}$. Then the Frenet formulae for the curve $\gamma$ are given by $([14,15])$

$$
\left(\begin{array}{c}
\mathrm{T}_{\gamma}^{\prime} \\
\mathrm{N}_{\gamma}^{\prime} \\
\mathrm{B}_{\gamma_{1}^{\prime}}^{\prime} \\
\mathrm{B}_{\gamma_{2}^{\prime}}^{\prime}
\end{array}\right)=\left(\begin{array}{cccc}
0 & \mathrm{~K}_{\gamma_{1}} & 0 & 0 \\
-\mathrm{K}_{\gamma 1} & 0 & \mathrm{~K}_{\gamma_{2}} & 0 \\
0 & -\mathrm{K}_{\gamma_{2}} & 0 & \mathrm{~K}_{\gamma_{3}} \\
0 & 0 & -\mathrm{K}_{\gamma_{3}} & 0
\end{array}\right)\left(\begin{array}{c}
\mathrm{T}_{\gamma} \\
\mathrm{N}_{\gamma} \\
\mathrm{B}_{\gamma_{1}} \\
\mathrm{~B}_{\gamma_{2}}
\end{array}\right) \text {. }
$$


From the above formulae, it follows that $\kappa_{\gamma_{3}} \not \equiv 0$ if and only if the curve $\gamma$ lies wholly in $\mathbb{E}^{4}$. This is equivalent to saying that $\kappa_{\gamma_{3}} \equiv 0$ if and only if the curve $\gamma$ lies wholly in a hypersurface in $\mathbb{E}^{4}$ (cf. $\left.[14,15]\right)$. We recall that the hypersurface in $\mathbb{E}^{4}$ defined by

$$
\mathbb{S}^{3}(1):=\left\{v \in \mathbb{E}^{4}:\langle v, v\rangle=1\right\}
$$

is called the unit-sphere with centre at the origin in $\mathbb{E}^{4}$. We also recall that the rectifying space of the curve $\gamma$ is the orthogonal complement $\mathrm{N}_{\gamma}{ }^{\perp}$ of its principal normal vector field $\mathrm{N}_{\gamma}$ defined by

$$
\mathrm{N}_{\gamma}{ }^{\perp}:=\left\{v \in \mathbb{E}^{4}:\left\langle v, \mathrm{~N}_{\gamma}\right\rangle=0\right\} .
$$

Consequently, $\mathrm{N}_{\gamma}{ }^{\perp}$ at each point $\gamma(\mathrm{s})$ on $\gamma$ is a three dimensional subspace of $\mathbb{E}^{4}$ spanned by $\left\{\mathrm{T}_{\gamma}(\mathrm{s}), \mathrm{B}_{\gamma_{1}}(\mathrm{~s}), \mathrm{B}_{\gamma_{2}}(\mathrm{~s})\right\}$.

\section{3 f-rectifying curves in $\mathbb{E}^{4}$}

As defined in [7], a unit-speed curve $\gamma: \mathrm{I} \longrightarrow \mathbb{E}^{4}$ (parametrized by arc length function s) is a rectifying curve if and only if its position vector always lies in its rectifying space, i.e., if and only if its position vector can be expressed as

$$
\gamma(\mathrm{s})=\lambda(\mathrm{s}) \mathrm{T}_{\gamma}(\mathrm{s})+\mu_{1}(\mathrm{~s}) \mathrm{B}_{\gamma_{1}}(\mathrm{~s})+\mu_{2}(\mathrm{~s}) \mathrm{B}_{\gamma_{2}}(\mathrm{~s})
$$

for some differentiable functions $\lambda, \mu_{1}, \mu_{2}: I \longrightarrow \mathbb{R}$ in parameter $s$, for each $s \in I$. Now, for some nowhere vanishing integrable function $f: I \longrightarrow \mathbb{R}$ in parameter $\mathrm{s}$, the $\mathrm{f}$-position vector of $\gamma$ in $\mathbb{E}^{4}$ is denoted and defined by

$$
\gamma_{f}(s):=\int f(s) d \gamma
$$

for all $s \in I$. Here the integral sign is used in the sense that $\gamma_{f}$ is an integral curve of the vector field $\mathrm{fT}_{\gamma}$ and after differentiating the previous equation we find $\gamma_{f}^{\prime}(s)=f(s) T_{\gamma}(s)$ for all $s \in I$. Keeping in mind this notion of position vector of a curve in $\mathbb{E}^{4}$, we define an f-rectifying curve in $\mathbb{E}^{4}$ as follows:

Definition 1 Let $\gamma: \mathrm{I} \longrightarrow \mathbb{E}^{4}$ be a unit-speed curve (parametrized by arc length function s) with Frenet apparatus $\left\{\mathrm{T}_{\gamma}, \mathrm{N}_{\gamma}, \mathrm{B}_{\gamma_{1}}, \mathrm{~B}_{\gamma_{2}}, \mathrm{~K}_{\gamma_{1}}, \mathrm{~K}_{\gamma_{2}}, \mathrm{~K}_{\gamma_{3}}\right\}$. Also, let $\mathrm{f}: \mathrm{I} \longrightarrow \mathbb{R}$ be a nowhere vanishing integrable function in parameter $\mathrm{s}$ with at least twice differentiable primitive function $\mathrm{F}$. Then $\gamma$ is called an f-rectifying 
curve in $\mathbb{E}^{4}$ if its $\mathrm{f}$-position vector $\gamma_{\mathrm{f}}$ always lies in its rectifying space in $\mathbb{E}^{4}$, i.e., if its $\mathrm{f}$-position vector $\gamma_{\mathrm{f}}$ in $\mathbb{E}^{4}$ can be expressed as

$$
\gamma_{\mathrm{f}}(\mathrm{s})=\lambda(\mathrm{s}) \mathrm{T}_{\gamma}(\mathrm{s})+\mu_{1}(\mathrm{~s}) \mathrm{B}_{\gamma_{1}}(\mathrm{~s})+\mu_{2}(\mathrm{~s}) \mathrm{B}_{\gamma_{2}}(\mathrm{~s})
$$

for all $s \in I$, where $\lambda, \mu_{1}, \mu_{2}: I \longrightarrow \mathbb{R}$ are three unique smooth functions in parameter $s$.

\section{Some geometric characterizations of f-rectifying curves in $\mathbb{E}^{4}$}

In this section, we characterize unit-speed f-rectifying curves in $\mathbb{E}^{4}$ in terms of their curvatures and components of their f-position vectors. First, in the following theorem, we establish a necessary as well as sufficient condition for a unit-speed curve in $\mathbb{E}^{4}$ to be an f-rectifying curve.

Theorem 1 Let $\gamma: \mathrm{I} \longrightarrow \mathbb{E}^{4}$ be a unit-speed curve (parametrized by arc length $\mathrm{s})$, having nowhere vanishing curvatures $\mathrm{\kappa}_{\gamma_{1}}, \mathrm{\kappa}_{\gamma_{2}}$ and $\mathrm{\kappa}_{\gamma_{3}}$. Also, let $\mathrm{f}: \mathrm{I} \longrightarrow \mathbb{R}$ be a nowhere vanishing integrable function in parameter $\mathrm{s}$ with at least twice differentiable primitive function $\mathrm{F}$. Then, up to isometries of $\mathbb{E}^{4}, \gamma$ is congruent to an f-rectifying curve in $\mathbb{E}^{4}$ if and only if the following equation is satisfied:

$$
\frac{d}{d s}\left(\frac{\frac{d}{d s}\left(\frac{\kappa_{\gamma_{1}}(s)}{\kappa_{\gamma_{2}}(s)} F(s)\right)}{\kappa_{\gamma_{3}}(s)}\right)+\frac{\kappa_{\gamma_{1}}(s) \kappa_{\gamma_{3}}(s)}{\kappa_{\gamma_{2}}(s)} F(s)=0
$$

for all $\mathrm{s} \in \mathrm{I}$.

Proof. Let us first assume that $\gamma: \mathrm{I} \longrightarrow \mathbb{E}^{4}$ be an f-rectifying curve having nowhere vanishing curvatures $\kappa_{\gamma_{1}}, \kappa_{\gamma_{2}}$ and $\kappa_{\gamma_{3}}$. Then for some differentiable functions $\lambda, \mu_{1}, \mu_{2}: I \longrightarrow \mathbb{R}$ in parameter $s$, its $f$-position vector $\gamma_{f}$ satisfies equation (2). Differentiating (2) and then applying (1), we obtain

$$
\begin{aligned}
f(s) T_{\gamma}(s)=\lambda^{\prime}(s) T_{\gamma}(s) & +\left(\lambda(s) \kappa_{\gamma_{1}}(s)-\mu_{1}(s) \kappa_{\gamma_{2}}(s)\right) N_{\gamma}(s) \\
+ & \left(\mu_{1}^{\prime}(s)-\mu_{2}(s) \kappa_{\gamma_{3}}(s)\right) B_{\gamma_{1}}(s) \\
+ & \left(\mu_{2}^{\prime}(s)+\mu_{1}(s) \kappa_{\gamma_{3}}(s)\right) B_{\gamma_{2}}(s)
\end{aligned}
$$

for all $s \in$ I. Equating the coefficients from both sides of (4), we get

$$
\left\{\begin{aligned}
\lambda^{\prime}(s) & =f(s), \\
\lambda(s) \kappa_{\gamma_{1}}(s)-\mu_{1}(s) \kappa_{\gamma_{2}}(s) & =0, \\
\mu_{1}^{\prime}(s)-\mu_{2}(s) \kappa_{\gamma_{3}}(s) & =0, \\
\mu_{2}^{\prime}(s)+\mu_{1}(s) \kappa_{\gamma_{3}}(s) & =0
\end{aligned}\right.
$$


for all $s \in$ I. From first three equations of (5), after some steps, we find

$$
\left\{\begin{aligned}
\lambda(s) & =F(s), \\
\mu_{1}(s) & =\frac{\kappa_{\gamma_{1}}(s)}{\kappa_{\gamma_{2}}(s)} F(s), \\
\mu_{2}(s) & =\frac{1}{\kappa_{\gamma_{3}}(s)} \frac{d}{d s}\left(\frac{\kappa_{\gamma_{1}}(s)}{\kappa_{\gamma_{2}}(s)} \mathrm{F}(s)\right)
\end{aligned}\right.
$$

for all $s \in$ I. Substituting (6) in the fourth one of (5), we obtain

$$
\frac{d}{d s}\left(\frac{\frac{d}{d s}\left(\frac{\kappa_{\gamma_{1}}(s)}{\kappa_{\gamma_{2}}(s)} F(s)\right)}{\kappa_{\gamma_{3}}(s)}\right)+\frac{\kappa_{\gamma_{1}}(s) \kappa_{\gamma_{3}}(s)}{\kappa_{\gamma_{2}}(s)} F(s)=0
$$

for all $s \in \mathrm{I}$.

Conversely, we assume that $\gamma: \mathrm{I} \longrightarrow \mathbb{E}^{4}$ is a unit-speed curve having nowhere vanishing curvatures $\kappa_{\gamma_{1}}, \kappa_{\gamma_{2}}$ and $\kappa_{\gamma_{3}}$, and $f: I \longrightarrow \mathbb{R}$ is a nowhere vanishing integrable function in parameter $s$ with at least twice differentiable primitive function $F$ such that the equation (3) is satisfied.

Let us define a vector field $\mathrm{V}$ along $\gamma$ by

$$
\begin{aligned}
V(s)=\gamma_{f}(s) & -F(s) T_{\gamma}(s)-\frac{\kappa_{\gamma_{1}}(s)}{\kappa_{\gamma_{2}}(s)} F(s) B_{\gamma_{1}}(s) \\
& -\frac{1}{\kappa_{\gamma_{3}}(s)} \frac{d}{d s}\left(\frac{\kappa_{\gamma_{1}}(s)}{\kappa_{\gamma_{2}}(s)} F(s)\right) B_{\gamma_{2}}(s)
\end{aligned}
$$

for all $s \in$ I. Differentiating (7) and then applying (1) and (3), we find that $V^{\prime}(s)=0$ for all $s \in I$. This implies that $V$ is constant along $\gamma$. Hence, up to isometries of $\mathbb{E}^{4}, \gamma$ is congruent to an f-rectifying curve in $\mathbb{E}^{4}$.

Remark 1 For an f-rectifying curve in $\mathbb{E}^{4}$, if all of its curvature functions $\kappa_{\gamma_{1}}, \kappa_{\gamma_{2}}$ and $\kappa_{\gamma_{3}}$ are non-zero constants, say, $\kappa_{\gamma_{1}}(\mathrm{~s})=\mathrm{a}_{1} \neq 0, \kappa_{\gamma_{2}}(\mathrm{~s})=\mathrm{a}_{2} \neq 0$ and $\mathrm{K}_{\gamma_{3}}(\mathrm{~s})=\mathrm{a}_{3} \neq 0$ for all $\mathrm{s} \in \mathrm{I}$, then from (3), we obtain

$$
F^{\prime \prime}(s)+a_{3}^{2} F(s)=0 .
$$

If $\mathrm{f}$ is non-zero constant or linear, then from (8) we find $\mathrm{a}_{3}=0$ which is a contradiction. Again, if $\mathrm{f}$ is non-linear, then from (8) we find $\mathrm{a}_{3}$ is nonconstant which is also a contradiction.

According to the above remark, we have the following theorem: 
Theorem 2 Let $\gamma: \mathrm{I} \longrightarrow \mathbb{E}^{4}$ be a unit-speed curve having nowhere vanishing curvatures $\mathrm{K}_{\gamma_{1}}, \mathrm{~K}_{\gamma_{2}}$ and $\mathrm{K}_{\gamma_{3}}$. Then $\gamma$ is not congruent to an $\mathrm{f}$-rectifying curve for any choice of $\mathrm{f}$ if and only if all of its curvatures $\mathrm{\kappa}_{\gamma_{1}}, \mathrm{\kappa}_{\gamma_{2}}$ and $\mathrm{\kappa}_{\gamma_{3}}$ are constants.

Now, it may happen that any two among the three nowhere vanishing curvatures $\kappa_{\gamma_{1}}, \kappa_{\gamma_{2}}$ and $\kappa_{\gamma_{3}}$ are constants. Then, as some immediate consequences of Theorem 1, the following theorem provides some characterizations regarding the non-constant one.

Theorem 3 Let $\gamma: \mathrm{I} \longrightarrow \mathbb{E}^{4}$ be a unit-speed curve (parametrized by arc length $\mathrm{s})$, having nowhere vanishing curvatures $\kappa_{\gamma_{1}}, \kappa_{\gamma_{2}}$ and $\kappa_{\gamma_{3}}$. Also, let $\mathrm{f}: \mathrm{I} \longrightarrow \mathbb{R}$ be a nowhere vanishing integrable function in parameter $\mathrm{s}$ with at least twice differentiable primitive function $\mathrm{F}$. We have the following:

(i) If the first curvature $\mathrm{K}_{\gamma_{1}}$ and the second curvature $\mathrm{K}_{\gamma_{2}}$ are constants, then $\gamma$ is congruent to an $\mathbf{f}$-rectifying curve in $\mathbb{E}^{4}$ if and only if the third curvature $\mathrm{\kappa}_{\gamma_{3}}$ satisfies the following differential equation:

$$
\kappa_{\gamma_{3}}(s) F^{\prime \prime}(s)-\kappa_{\gamma_{3}}^{\prime}(s) F^{\prime}(s)+\kappa_{\gamma_{3}}^{3}(s) F(s)=0 .
$$

(ii) If the first curvature $\mathrm{K}_{\gamma_{1}}$ and the third curvature $\mathrm{K}_{\gamma_{3}}(=\mathrm{a})$ are constants, then $\gamma$ is congruent to an $\mathbf{f}$-rectifying curve in $\mathbb{E}^{4}$ if and only if the second curvature $\mathrm{K}_{\gamma_{2}}$ satisfies the following differential equation:

$$
\frac{d^{2}}{d s^{2}}\left(\frac{F(s)}{K_{\gamma_{2}}(s)}\right)+a^{2} \frac{F(s)}{K_{\gamma_{2}}(s)}=0 .
$$

(iii) If the second curvature $\mathrm{\kappa}_{\gamma_{2}}$ and the third curvature $\mathrm{\kappa}_{\gamma_{3}}(=\mathrm{a})$ are constants, then $\gamma$ is congruent to an f-rectifying curve in $\mathbb{E}^{4}$ if and only if the first curvature $\mathrm{K}_{\gamma_{1}}$ satisfies the following differential equation:

$$
\frac{d^{2}}{d s^{2}}\left(\kappa_{\gamma_{1}}(s) F(s)\right)+a^{2} \kappa_{\gamma 1}(s) F(s)=0 .
$$

Analogous characterizations can be derived as consequences of Theorem 1 when any one of $\kappa_{\gamma_{1}}, \kappa_{\gamma_{2}}$ or $\kappa_{\gamma_{3}}$ is a constant.

Next, in the following theorem, we characterize unit-speed f-rectifying curves in $\mathbb{E}^{4}$ in terms of norm functions, tangential, normal, first and second binormal components of their f-position vectors. 
Theorem 4 Let $\gamma: \mathrm{I} \longrightarrow \mathbb{E}^{4}$ be a unit-speed curve (parametrized by arc length $\mathrm{s})$, having nowhere vanishing curvatures $\mathrm{\kappa}_{\gamma_{1}}, \mathrm{\kappa}_{\gamma_{2}}$ and $\mathrm{\kappa}_{\gamma_{3}}$. Also, let $\mathrm{f}: \mathrm{I} \longrightarrow \mathbb{R}$ be a nowhere vanishing integrable function in parameter $\mathrm{s}$ with at least twice differentiable primitive function $\mathrm{F}$. If $\gamma$ is an f-rectifying curve in $\mathbb{E}^{4}$, then the following statements are true:

(i) The norm function $\rho=\left\|\gamma_{\mathrm{f}}\right\|$ is given by

$$
\rho(s)=\sqrt{F^{2}(s)+c^{2}}
$$

for all $\mathrm{s} \in \mathrm{I}$, where $\mathrm{c}$ is a non-zero constant.

(ii) The tangential component $\left\langle\gamma_{\mathrm{f}}, \mathrm{T}_{\gamma}\right\rangle$ of the $\mathrm{f}$-position vector $\gamma_{\mathrm{f}}$ of $\gamma$ is given by

$$
\left\langle\gamma_{f}(s), T_{\gamma}(s)\right\rangle=F(s)
$$

for all $\mathrm{s} \in \mathrm{I}$.

(iii) The normal component $\gamma_{f}^{\mathrm{N}_{\gamma}}$ of the f-position vector $\gamma_{\mathrm{f}}$ of $\gamma$ has constant length and the norm function $\rho$ is non-constant.

(iv) The first binormal component $\left\langle\gamma_{\mathrm{f}}, \mathrm{B}_{\gamma_{1}}\right\rangle$ and the second binormal component $\left\langle\gamma_{f}, B_{\gamma_{2}}\right\rangle$ of the $\mathrm{f}$-position vector $\gamma_{f}$ of $\gamma$ are respectively given by

$$
\begin{aligned}
& \left\langle\gamma_{\mathrm{f}}(s), B_{\gamma_{1}}(s)\right\rangle=\frac{\kappa_{\gamma_{1}}(s)}{\kappa_{\gamma_{2}}(s)} \mathrm{F}(\mathrm{s}), \\
& \left\langle\gamma_{\mathrm{f}}(s), B_{\gamma_{2}}(s)\right\rangle=\frac{1}{\kappa_{\gamma_{3}}(s)} \frac{\mathrm{d}}{\mathrm{ds}}\left(\frac{\kappa_{\gamma_{1}}(s)}{\kappa_{\gamma_{2}}(s)} \mathrm{F}(s)\right)
\end{aligned}
$$

for all $\mathrm{s} \in \mathrm{I}$.

Conversely, if $\gamma: \mathrm{I} \longrightarrow \mathbb{E}^{4}$ is a unit-speed curve (parametrized by arc length $\mathrm{s})$, having nowhere vanishing curvatures $\mathrm{\kappa}_{\gamma_{1}}, \mathrm{\kappa}_{\gamma_{2}}$ and $\mathrm{\kappa}_{\gamma_{3}}$, and $\mathrm{f}: \mathrm{I} \longrightarrow \mathbb{R}$ is a nowhere vanishing integrable function in parameter $\mathrm{s}$ with at least twice differentiable primitive function $\mathrm{F}$ such that any one of the statements (i), (ii), (iii) or (iv) is true, then $\gamma$ is an f-rectifying curve in $\mathbb{E}^{4}$.

Proof. Let us first assume that $\gamma: \mathrm{I} \longrightarrow \mathbb{E}^{4}$ is an f-rectifying curve having nowhere vanishing curvatures $\kappa_{\gamma_{1}}, \kappa_{\gamma_{2}}$ and $\kappa_{\gamma_{3}}$. Then for some differentiable functions $\lambda, \mu_{1}, \mu_{2}: I \longrightarrow \mathbb{R}$ in parameter $s$, the f-position vector $\gamma_{f}$ of the 
curve $\gamma$ in $\mathbb{E}^{4}$ satisfies equation (2) and from the proof of Theorem 1, we have (5) and (6). Now, from last two equations of (5), we easily find

$$
\mu_{1}(s) \mu_{1}^{\prime}(s)+\mu_{2}(s) \mu_{2}^{\prime}(s)=0
$$

for all $s \in$ I. Integrating previous equation, we obtain

$$
\mu_{1}^{2}(s)+\mu_{2}^{2}(s)=c^{2}
$$

for all $s \in I$, where $c$ is an arbitrary non-zero constant. We have the following: (i) Using (2), (6) and (9), the norm function $\rho=\left\|\gamma_{\mathrm{f}}\right\|$ is given by

$$
\rho^{2}(s)=\left\|\gamma_{f}(s)\right\|^{2}=\left\langle\gamma_{f}(s), \gamma_{f}(s)\right\rangle=F^{2}(s)+c^{2},
$$

i.e.,

$$
\rho(s)=\sqrt{F^{2}(s)+c^{2}}
$$

for all $s \in \mathrm{I}$, where $\mathrm{c}$ is a non-zero constant.

(ii) Using (2) and (6), the tangential component $\left\langle\gamma_{\mathrm{f}}, \mathrm{T}_{\gamma}\right\rangle$ of $\gamma_{\mathrm{f}}$ is given by

$$
\left\langle\gamma_{\mathrm{f}}(\mathrm{s}), \mathrm{T}_{\gamma}(\mathrm{s})\right\rangle=\lambda(\mathrm{s})=\mathrm{F}(\mathrm{s})
$$

for all $s \in I$.

(iii) An f-position vector $\alpha_{\mathrm{f}}$ of an arbitrary curve $\alpha: \mathrm{J} \longrightarrow \mathbb{E}^{4}$ can be decomposed as

$$
\alpha_{f}(t)=v(t) T_{\gamma}(t)+\alpha_{f}^{N_{\gamma}}(t), \quad t \in J,
$$

for some differentiable function $v: \mathrm{I} \longrightarrow \mathbb{R}$, where $\alpha_{\mathrm{f}}^{\mathrm{N}_{\gamma}}$ denotes the normal component of $\alpha_{\mathrm{f}}$. Here, $\gamma$ is an f-rectifying curve in $\mathbb{E}^{4}$ and hence from (2), it is evident that the normal component $\gamma_{f}^{N_{\gamma}}$ of $\gamma_{f}$ is given by

$$
\gamma_{f}^{N_{\gamma}}(s)=\mu_{1}(s) B_{\gamma_{1}}(s)+\mu_{2}(s) B_{\gamma_{2}}(s)
$$

for all $s \in \mathrm{I}$. Therefore, we have

$$
\left\|\gamma_{f}^{N_{\gamma}}(s)\right\|=\sqrt{\left\langle\gamma_{f}^{N_{\gamma}}(s), \gamma_{f}^{N_{\gamma}}(s)\right\rangle}=\sqrt{\mu_{1}^{2}(s)+\mu_{2}^{2}(s)}
$$

for all $s \in$ I. Now, by using (9), we see that $\left\|\gamma_{f}^{N_{\gamma}}(s)\right\|=c$. This implies that $\gamma_{f}^{N_{\gamma}}$ has constant length. Furthermore, from statement (i), it follows that the norm function $\rho=\left\|\gamma_{\mathrm{f}}\right\|$ is non-constant. 
(iv) Using (2) and (6), the first binormal component $\left\langle\gamma_{f}, B_{\gamma_{1}}\right\rangle$ of $\gamma_{f}$ is given by

$$
\left\langle\gamma_{\mathrm{f}}(\mathrm{s}), \mathrm{B}_{\gamma_{1}}(\mathrm{~s})\right\rangle=\mu_{1}(\mathrm{~s})=\frac{\kappa_{\gamma_{1}}(\mathrm{~s})}{\kappa_{\gamma_{2}}(\mathrm{~s})} \mathrm{F}(\mathrm{s})
$$

for all $s \in I$, and the second binormal component $\left\langle\gamma_{f}, B_{\gamma_{2}}\right\rangle$ of $\gamma_{f}$ is given by

$$
\left\langle\gamma_{\mathrm{f}}(\mathrm{s}), \mathrm{B}_{\gamma_{2}}(\mathrm{~s})\right\rangle=\mu_{2}(\mathrm{~s})=\frac{1}{\kappa_{\gamma_{3}}(\mathrm{~s})} \frac{\mathrm{d}}{\mathrm{ds}}\left(\frac{\mathrm{K}_{\gamma_{1}}(\mathrm{~s})}{\kappa_{\gamma_{2}}(\mathrm{~s})} \mathrm{F}(\mathrm{s})\right)
$$

for all $s \in \mathrm{I}$.

Conversely, we assume that $\gamma: \mathrm{I} \longrightarrow \mathbb{E}^{4}$ is a unit-speed curve having nowhere vanishing curvatures $\kappa_{\gamma_{1}}, \kappa_{\gamma_{2}}$ and $\kappa_{\gamma_{3}}$, and $f: I \longrightarrow \mathbb{R}$ is a nowhere vanishing integrable function in parameter $s$ with at least twice differentiable primitive function F such that statement (i) or statement (ii) is true. For statement (i), we have

$$
\left\langle\gamma_{\mathrm{f}}(\mathrm{s}), \gamma_{\mathrm{f}}(\mathrm{s})\right\rangle=\mathrm{F}^{2}(\mathrm{~s})+\mathrm{c}^{2}
$$

for all $s \in I$, where $c$ is a non-zero constant. On differentiation of last equation, we obtain

$$
\left\langle\gamma_{\mathrm{f}}(\mathrm{s}), \mathrm{T}_{\gamma}(\mathrm{s})\right\rangle=\mathrm{F}(\mathrm{s})
$$

for all $s \in$ I. So in either case we have equation (10). Differentiating (10) and by using (1), we finally obtain

$$
\left\langle\gamma_{\mathrm{f}}(\mathrm{s}), \mathrm{N}_{\gamma}(\mathrm{s})\right\rangle=0
$$

for all $s \in I$. This asserts us that $\gamma$ is an f-rectifying curve in $\mathbb{E}^{4}$.

Next, we assume that statement (iii) is true. Then $\left\|\gamma_{f}^{N_{\gamma}}\right\|=c$, say. Now, the normal component $\gamma_{f}^{N_{\gamma}}$ is given by

$$
\gamma_{f}(s)=F(s) T_{\gamma}(s)+\gamma_{f}^{N_{\gamma}}(s)
$$

for all $s \in \mathrm{I}$. Therefore, we have

$$
\left\langle\gamma_{\mathrm{f}}(\mathrm{s}), \gamma_{\mathrm{f}}(\mathrm{s})\right\rangle=\left\langle\gamma_{\mathrm{f}}(\mathrm{s}), \mathrm{T}_{\gamma}(\mathrm{s})\right\rangle^{2}+\mathrm{c}^{2}
$$

for all $s \in$ I. Differentiating previous equation and using (1), we obtain

$$
\left\langle\gamma_{\mathrm{f}}(\mathrm{s}), \mathrm{N}_{\gamma}(\mathrm{s})\right\rangle=0
$$

for all $s \in \mathrm{I}$. This implies that $\gamma$ is an f-rectifying curve in $\mathbb{E}^{4}$. 
Finally, we assume that statement (iv) is true. Then we have

$$
\begin{aligned}
\left\langle\gamma_{f}(s), B_{\gamma_{1}}(s)\right\rangle & =\frac{\kappa_{\gamma_{1}}(s)}{\kappa_{\gamma_{2}}(s)} F(s), \\
\left\langle\gamma_{f}(s), B_{\gamma_{2}}(s)\right\rangle & =\frac{1}{\kappa_{\gamma_{3}}(s)} \frac{d}{d s}\left(\frac{\kappa_{\gamma_{1}}(s)}{\kappa_{\gamma_{2}}(s)} F(s)\right)
\end{aligned}
$$

for all $s \in$ I. Differentiating (11) and using (1), we obtain

$$
-\kappa_{\gamma_{2}}(s)\left\langle\gamma_{f}(s), N_{\gamma}(s)\right\rangle+\kappa_{\gamma_{3}}(s)\left\langle\gamma_{f}(s), B_{\gamma_{2}}(s)\right\rangle=\frac{d}{d s}\left(\frac{\kappa_{\gamma_{1}}(s)}{\kappa_{\gamma_{2}}(s)} F(s)\right)
$$

for all $s \in$ I. From the equations (12) and last equation, we find

$$
\left\langle\gamma_{\mathrm{f}}(\mathrm{s}), \mathrm{N}_{\gamma}(\mathrm{s})\right\rangle=0
$$

for all $s \in \mathrm{I}$. Consequently, $\gamma$ is an f-rectifying curve in $\mathbb{E}^{4}$.

\section{Classification of f-rectifying curves in $\mathbb{E}^{4}$}

In many papers, e.g., $[3,7,8,9]$, several interesting results were found primarily attempting towards the classification of the rectifying curves which are mostly based on their parametrizations. In this section we attempt for the same in the case of unit-speed f-rectifying curves in $\mathbb{E}^{4}$ but this classification is totally based on the parametrizations of their f-position vectors.

Theorem 5 Let $\gamma: \mathrm{I} \longrightarrow \mathbb{E}^{4}$ be a unit-speed curve (parametrized by arc length s) having nowhere vanishing curvatures $\kappa_{\gamma_{1}}, \kappa_{\gamma_{2}}$ and $\kappa_{\gamma_{3}}$, and let $\mathrm{f}: \mathrm{I} \longrightarrow \mathbb{R}$ be a nowhere vanishing integrable function in parameter $\mathrm{s}$ with at least twice differentiable primitive function $\mathrm{F}$. Then $\gamma$ is an $\mathrm{f}$-rectifying curve in $\mathbb{E}^{4}$ if and only if, up to a parametrization, its $\mathrm{f}$-position vector $\gamma_{\mathrm{f}}$ is given by

$$
\gamma_{\mathrm{f}}(\mathrm{t})=\frac{\mathrm{c}}{\cos \left(\mathrm{t}+\arctan \left(\frac{\mathrm{F}\left(\mathrm{s}_{\mathrm{o}}\right)}{\mathrm{c}}\right)\right)} \alpha(\mathrm{t})
$$

for all $\mathrm{t} \in \mathrm{J}$, where $\mathrm{c}$ is a positive constant, $\mathrm{s}_{0} \in \mathrm{I}$ and $\alpha: \mathrm{J} \longrightarrow \mathbb{S}^{3}(1)$ is a unit-speed curve having $\mathrm{t}: \mathrm{I} \longrightarrow \mathrm{J}$ as arc length function based at $\mathrm{s}_{0}$.

Proof. Let us first assume that $\gamma: \mathrm{I} \longrightarrow \mathbb{E}^{4}$ be an f-rectifying curve having nowhere vanishing curvatures $\kappa_{\gamma_{1}}, \kappa_{\gamma_{2}}$ and $\kappa_{\gamma_{3}}$. Then from the proof of Theorem 4, we have

$$
\rho(s)=\sqrt{F^{2}(s)+c^{2}}
$$


for all $s \in I$, where we may choose $c$ as a positive real constant. Now, we define a new curve $\alpha$ in $\mathbb{E}^{4}$ by

$$
\alpha(s):=\frac{1}{\rho(s)} \gamma_{f}(s)
$$

for all $s \in \mathrm{I}$. Then we find

$$
\langle\alpha(s), \alpha(s)\rangle=1
$$

for all $s \in \mathrm{I}$. Therefore, $\alpha$ is a curve whose trace is lying wholly in the unit sphere $\mathbb{S}^{3}(1)$. Differentiating (16), we get

$$
\left\langle\alpha(s), \alpha^{\prime}(s)\right\rangle=0,
$$

for all $s \in I$. Now, from (14) and (15), we have

$$
\gamma_{\mathrm{f}}(\mathrm{s})=\alpha(\mathrm{s}) \sqrt{\mathrm{F}^{2}(\mathrm{~s})+\mathrm{c}^{2}}
$$

for all $s \in$ I. Differentiating (18), we find

$$
f(s) T_{\gamma}(s)=\alpha^{\prime}(s) \sqrt{F^{2}(s)+c^{2}}+\frac{\alpha(s) f(s) F(s)}{\sqrt{F^{2}(s)+c^{2}}}
$$

for all $s \in \mathrm{I}$. Using (16), (17) and (19), we obtain

$$
\left\langle\alpha^{\prime}(s), \alpha^{\prime}(s)\right\rangle=\frac{c^{2} f^{2}(s)}{\left(F^{2}(s)+c^{2}\right)^{2}}
$$

for all $s \in \mathrm{I}$. Therefore, we get

$$
\left\|\alpha^{\prime}(s)\right\|=\sqrt{\left\langle\alpha^{\prime}(s), \alpha^{\prime}(s)\right\rangle}=\frac{c f(s)}{F^{2}(s)+c^{2}}
$$

for all $s \in I$. Let $s_{0} \in I$ and $t: I \longrightarrow J$ be arc length function of $\alpha$ based at $s_{0}$ given by

$$
\mathrm{t}=\int_{\mathrm{s}_{0}}^{\mathrm{s}}\left\|\alpha^{\prime}(\mathrm{u})\right\| \mathrm{du} .
$$

Then we find

$$
\begin{aligned}
\mathrm{t} & =\int_{s_{0}}^{\mathrm{s}} \frac{\mathrm{cf}(\mathrm{u})}{\mathrm{F}^{2}(\mathrm{u})+\mathrm{c}^{2}} d \mathrm{u} \\
\Longrightarrow \quad \mathrm{t} & =\arctan \left(\frac{\mathrm{F}(\mathrm{s})}{\mathrm{c}}\right)-\arctan \left(\frac{\mathrm{F}\left(\mathrm{s}_{0}\right)}{\mathrm{c}}\right)
\end{aligned}
$$




$$
\Longrightarrow \mathrm{F}(\mathrm{s})=\mathrm{ctan}\left(\mathrm{t}+\arctan \left(\frac{\mathrm{F}\left(\mathrm{s}_{0}\right)}{\mathrm{c}}\right)\right) \text {. }
$$

Substituting previous equation in (18), we obtain the f-position vector of $\gamma$ as follows:

$$
\gamma_{\mathrm{f}}(\mathrm{t})=\frac{\mathrm{c}}{\cos \left(\mathrm{t}+\arctan \left(\frac{\mathrm{F}\left(\mathrm{s}_{0}\right)}{\mathrm{c}}\right)\right)} \alpha(\mathrm{t})
$$

for all $t \in J$.

Conversely, let $\gamma$ be a curve in $\mathbb{E}^{4}$ such that its f-position vector $\gamma_{\mathrm{f}}$ is given by (13), where $c$ is a positive constant, $s_{0} \in I$ and $\alpha: J \longrightarrow \mathbb{S}^{3}(1)$ is a unit-speed curve having $\mathrm{t}: \mathrm{I} \longrightarrow \mathrm{J}$ as arc length function based at $\mathrm{s}_{0}$. Differentiating (13), we obtain

$$
\gamma_{f}^{\prime}(t)=\frac{c \sin \left(t+\arctan \left(\frac{F\left(s_{0}\right)}{c}\right)\right)}{\cos ^{2}\left(t+\arctan \left(\frac{F\left(s_{0}\right)}{c}\right)\right)} \alpha(t)+\frac{c}{\cos \left(t+\arctan \left(\frac{F\left(s_{0}\right)}{c}\right)\right)} \alpha^{\prime}(t)
$$

for all $t \in J$. Since $\alpha$ is a unit-speed curve in the unit-sphere $\mathbb{S}^{3}(1)$, we have $\left\langle\alpha^{\prime}(\mathrm{t}), \alpha^{\prime}(\mathrm{t})\right\rangle=1,\langle\alpha(\mathrm{t}), \alpha(\mathrm{t})\rangle=1$ and consequently $\left\langle\alpha(\mathrm{t}), \alpha^{\prime}(\mathrm{t})\right\rangle=0$ for all $\mathrm{t} \in \mathrm{J}$. Therefore, from (13) and (20), we have

$$
\left\{\begin{array}{l}
\left\langle\gamma_{f}(t), \gamma_{f}(t)\right\rangle=\frac{c^{2}}{\cos ^{2}\left(t+\arctan \left(\frac{F\left(s_{0}\right)}{c}\right)\right)}, \\
\left\langle\gamma_{f}(t), \gamma_{f}^{\prime}(t)\right\rangle=\frac{c^{2} \sin \left(t+\arctan \left(\frac{F\left(s_{0}\right)}{c}\right)\right)}{\cos ^{3}\left(t+\arctan \left(\frac{F\left(s_{0}\right)}{c}\right)\right)}, \\
\left\langle\gamma_{f}^{\prime}(t), \gamma_{f}^{\prime}(t)\right\rangle=\frac{c^{2}}{\cos ^{4}\left(t+\arctan \left(\frac{F\left(s_{0}\right)}{c}\right)\right)}
\end{array}\right.
$$

for all $t \in J$. We may reparametrize $\gamma$ by

$$
\mathrm{t}=\arctan \left(\frac{\mathrm{F}(\mathrm{s})}{\mathrm{c}}\right)-\arctan \left(\frac{\mathrm{F}\left(\mathrm{s}_{0}\right)}{\mathrm{c}}\right) .
$$


Then $s$ becomes arc length parameter of $\gamma$ and equations (21) reduce to

$$
\left\{\begin{array}{l}
\left\langle\gamma_{\mathrm{f}}(\mathrm{s}), \gamma_{\mathrm{f}}(\mathrm{s})\right\rangle=\frac{\mathrm{c}^{2}}{\cos ^{2}\left(\arctan \left(\frac{\mathrm{F}(\mathrm{s})}{\mathrm{c}}\right)\right)}, \\
\left\langle\gamma_{\mathrm{f}}(\mathrm{s}), \gamma_{\mathrm{f}}^{\prime}(\mathrm{s})\right\rangle=\frac{\mathrm{c}^{2} \sin \left(\arctan \left(\frac{\mathrm{F}(\mathrm{s})}{\mathrm{c}}\right)\right)}{\cos ^{3}\left(\arctan \left(\frac{\mathrm{F}(\mathrm{s})}{\mathrm{c}}\right)\right)}, \\
\left\langle\gamma_{\mathrm{f}}^{\prime}(\mathrm{s}), \gamma_{\mathrm{f}}^{\prime}(\mathrm{s})\right\rangle=\frac{\mathrm{c}^{2}}{\cos ^{4}\left(\arctan \left(\frac{\mathrm{F}(\mathrm{s})}{\mathrm{c}}\right)\right)}
\end{array}\right.
$$

for all $s \in$ I. Now, the normal component $\gamma_{f}^{N_{\gamma}}$ of $\gamma_{f}$ is given by

$$
\left\langle\gamma_{f}^{N_{\gamma}}(s), \gamma_{f}^{N_{\gamma}}(s)\right\rangle=\left\langle\gamma_{f}(s), \gamma_{f}(s)\right\rangle-\frac{\left\langle\gamma_{f}(s), \gamma_{f}^{\prime}(s)\right\rangle^{2}}{\left\langle\gamma_{f}^{\prime}(s), \gamma_{f}^{\prime}(s)\right\rangle}
$$

for all $s \in \mathrm{I}$. Then substituting (22) in last equation, we obtain

$$
g\left(\gamma_{f}^{N_{\gamma}}(s), \gamma_{f}^{N_{\gamma}}(s)\right)=\left\|\gamma_{f}^{N_{\gamma}}(s)\right\|^{2}=c^{2}
$$

for all $s \in$ I. This implies that $\gamma_{f}^{N_{\gamma}}$ has a constant length. Also, the norm function $\rho$ is given by

$$
\rho(s)=\sqrt{g\left(\gamma_{\mathrm{f}}(\mathrm{s}), \gamma_{\mathrm{f}}(\mathrm{s})\right)}=\frac{\mathrm{c}}{\cos \left(\arctan \left(\frac{\mathrm{F}(\mathrm{s})}{\mathrm{c}}\right)\right)}
$$

for all $\mathrm{s} \in \mathrm{I}$, and it is non-constant. Hence, by applying Theorem 4, we conclude that $\gamma$ is an f-rectifying curve in $\mathbb{E}^{4}$.

Finally, we cite an example of an f-rectifying curve lying wholly in $\mathbb{E}^{4}$.

Example 1 Let $\gamma$ be a unit-speed curve (parametrized by arc length s) in $\mathbb{E}^{4}$. Let $f$ be a nowhere vanishing integrable function in parameter $s$ defined by

$$
f(s):=\exp s .
$$

Then its primitive function $F$ is given by

$$
\mathrm{F}(\mathrm{s})=\exp \mathrm{s}+\mathrm{c}_{1},
$$


where $c_{1}$ is an arbitrary constant. We choose $c_{1}=0$ and substitute

$$
\mathrm{F}(\mathrm{s})=\tan \left(\mathrm{t}+\arctan \left(\frac{\mathrm{F}(0)}{1}\right)\right)=\tan \left(\mathrm{t}+\frac{\pi}{4}\right),
$$

i.e.,

$$
s=\ln \left|\tan \left(t+\frac{\pi}{4}\right)\right| .
$$

Now, let, up to a parametrization, the f-position vector $\gamma_{\mathrm{f}}$ of $\gamma$ is given by

$$
\gamma_{\mathrm{f}}(\mathrm{t})=\frac{1}{\cos \left(\mathrm{t}+\frac{\pi}{4}\right)} \alpha(\mathrm{t})
$$

where $\alpha$ be a curve in $\mathbb{E}^{4}$ defined by

$$
\alpha(t):=\frac{1}{\sqrt{2}}(\sin t, \cos t, \sin t, \cos t) .
$$

Evidently, we have $\langle\alpha(t), \alpha(t)\rangle=1$ and $\left\langle\alpha^{\prime}(t), \alpha^{\prime}(t)\right\rangle=1$ for all $t$. Therefore, $\alpha$ is a unit-speed curve in $\mathbb{S}^{3}(1)$ having $t$ as arc length function based at 0 . Consequently, $\gamma$ is an f-rectifying curve and, up to a parametrization, it is given by

$$
\gamma(t)=\frac{1}{2}\left(\ln \left|\frac{1+\sin 2 t}{\cos 2 t}\right|, \ln \left|\frac{1-\sin 2 t}{\cos 2 t}\right|, \ln \left|\frac{1+\sin 2 t}{\cos 2 t}\right|, \ln \left|\frac{1-\sin 2 t}{\cos 2 t}\right|\right) .
$$

Note: Examples of curves in $\mathbb{E}^{4}$ which are not f-rectifying for any choice of $f$ are trivial and can be easily constructed by violating the condition stated in Theorem 1. For example, according to Theorem 2 (which is an immediate consequence of Theorem 1), curves in $\mathbb{E}^{4}$ having non-zero constant first, second and third curvatures are not f-rectifying.

\section{Acknowledgements}

We would like to express our heartiest gratitude to the anonymous honourable referees for their valuable time and effort dedicated towards betterment of this article and for their pearls of comments and suggestions which helped to improve this article. 


\section{References}

[1] M. P. do Carmo, Differential Geometry of Curves and Surfaces, Revised and Updated Second Edition, Dover Publications, Inc., Mineola, New York, 2016.

[2] A. Pressley, Elementary Differential Geometry, Second Edition, SpringerVerlag, London, 2010.

[3] B. Y. Chen, When does the position vector of a space curve always lie in its rectifying plane? Amer. Math. Monthly, 110 (2003), 147-152.

[4] B. Y. Chen, Rectifying curves and geodesics on a cone in the Euclidean 3-space, Tamkang Jour. of Math., 48 (2017), 209-214.

[5] B. Y. Chen, F. Dillen, Rectifying curves as centrodes and extremal curves, Bull. Inst. Math. Acad. Sinica, 33 (2005), 77-90.

[6] S. Deshmukh, B. Y. Chen, S. Alshamari, On rectifying curves in Euclidean 3-space, Turk. J. Math., 42 (2018), 609-620.

[7] K. Ilarslan, E. Nesovic, Some characterizations of rectifying curves in the euclidean space $\mathbf{E}^{4}$, Turk. J. Math., 32(1) (2008), 21-30.

[8] S. Cambie, W. Goemans, I. V. Bussche, Rectifying curves in the ndimensional euclidean space, Turk. J. Math. 40(1) (2016), 210-223.

[9] K. Ilarslan, E. Nešović, T. M. Petrović, Some characterization of rectifying curves in the Minkowski 3-Space, Novi. Sad. Jour. Math., 33 (2003), 2332 .

[10] K. Ilarslan, E. Nešović, On rectifying curves as centrodes and extremal curves in the Minkowski 3-Space $\mathbb{E}_{1}^{3}$, Novi. Sad. Jour. Math., 37 (2007), $53-64$.

[11] Z. Iqbal, J. Sengupta, Non-null (spacelike or timelike) f-rectifying curves in the Minkowski 3-space $\mathbb{E}_{1}^{3}$, Eurasian Bul. Math., 3 (1) (2020), 38-55.

[12] Z. Iqbal, J. Sengupta, Null (lightlike) f-rectifying curves in the Minkowski 3-space $\mathbb{E}_{1}^{3}$, Fundam. J. Math. Appl. 3 (1) (2020), 8-16.

[13] Z. Iqbal, J. Sengupta, Differential geometric aspects of lightlike frectifying curves in Minkowski space-time, Diff. Geom. - Dyn. Syst., 22 (2020), 113-129. 
[14] H. Gluck, Higher curvatures of curves in euclidean space, Amer. Math. Mon. 73(7) (1966), 699-704.

[15] H. Gluck, Higher curvatures of curves in euclidean space, ii, Amer. Math. Mon. 74(9) (1967), 1049-1056.

Received: June 27, 2020 